\title{
Monosomy 9p
}

INSERM

\section{Source}

INSERM. (1999). Orphanet: an online rare disease and orphan drug data base.

Monosomy 9p. ORPHA:261112

Monosomy $9 \mathrm{p}$ is a rare chromosomal anomaly characterized by psychomotor

developmental delay, facial dysmorphism (trigonocephaly, midface hypoplasia, upslanting palpebral fissures, dysplastic small ears, flat nasal bridge with anteverted nostrils and long philtrum, micrognathia, choanal atresia, short neck), single umbilical artery, omphalocele, inguinal or umbilical hernia, genital abnormalities (hypospadia, cryptorchidism), muscular hypotonia and scoliosis. 